\title{
Matrix Scan: A Switch aided screen traversal mechanism for motor disabled
}

\author{
Rajat Yadav \\ SmartPhone Platform Team, \\ Samsung R\&D Institute, Bangalore, \\ India \\ rajat.yadav@samsung.com
}

\author{
Sameer Namdeo \\ SmartPhone Platform Team, \\ Samsung R\&D Institute, Bangalore, \\ India \\ sameer.nam@samsung.com
}

\author{
Kusumakar Dwivedi \\ SmartPhone Platform Team, \\ Samsung R\&D Institute, Bangalore, \\ India \\ k.dwivedi@samsung.com
}

\begin{abstract}
With the advancement in touch based devices these days, new and better options are coming to the fore every day. The increasing penetration of touch-based smart devices into the lives of normal users is an excellent example to portray the importance of such technology among varied user groups. However, the biggest challenge still is to make them cater the needs of everyone and use of latest technology should be possible irrespective of their varying physical abilities. In this proposal the focus is on motor disabled people who do not have much control on their limbs and free movement is very difficult for them. And hence the touch based interface is not a comfortable option for such users, since interacting with such devices requires performing gestures and actions on screen. To meet this challenge a grid-based screen traversal technique is suggested in this paper, which does not require direct contact with interface, but can be controlled using an external switch and minimal movement of limbs.
\end{abstract}

Keywords - Switch based access, motor disabled, tetraplegia, screen traversal, co-ordinate quantization, accessibility, humancomputer interaction, matrix scan.

\section{INTRODUCTION}

Over the time touch based devices have evolved from a mere accessory to an indispensable requirement of daily life. These devices rely heavily on touch interaction from user to accept input and often for performing a specific function the input is required at specific location on screen which renders a large section of user void of the innumerable advantages and benefits offered by such devices. This paper focuses on one such segment of users, i.e., motor disabled. Such users have impairments that limit the physical functioning of one or more limbs and hence a free and precise interaction with touch screen devices is a challenging task [1], [2]. Some of the major conditions that lead to the restricted movement of limbs are Parkinson's disease, Multiple Sclerosis, Cerebral Palsy, Muscular Dystrophy, Spina Bifida, ALS (Lou Gehrig's disease) and Essential Tremor (ET).

Devices such as external joysticks, Bluetooth keyboards and mouse, are available as additional alternate input options but they are of little assistance to the motor disabled users as they require significant movement from user to point and perform actions on screen, and hence do not offer complete convenience to motor disabled users.
There are few existing works which contribute towards making touch screen based interactive devices accessible to motor disabled users. Laser pointer method enables user to interact with touch screen devices without actually touching the device [3], [4]. But this approach has one limitation that it demands precision and hand stability from user, and does not offers easy access for target users due to hand jitters and lack of motor stability. Another track being followed for increasing accessibility of touch screen devices involves Eye-movement tracking [5]. This facilitates pointing and locating a screen element just by gazing on to it, and then issuing a command by performing a fixed gesture, like blink of an eye. This approach has three major limitations as compared to other methods. First, unlike mouse or other input devices, movement of eye is often involuntary and therefore it is difficult to control eye position continuously. The eye focus keeps shifting constantly and therefore, every such movement cannot be considered as a legitimate input. Second, there is no consistent method to confirm input from the input device, as there is with clicking a button on mouse, or in this case a simple switch. Using blink of eye for confirming an input returns unsatisfactory results and can cause unwanted inputs. And finally, the accuracy of available eye-tracking equipment is far less consistent than other manual input devices such as mouse [6].

Another work is Barrier Pointing [7], [8], which proposes a solution for people with restricted and unstable hand movement and it involves guiding the stylus along elevated physical edges around the screen to improve pointing accuracy [7]. Even though the solution seems elegant but it requires hardware changes and user interface redesign to align the elements at the edges.

Item scanning is another approach which can be used to traverse through elements in cases where item hierarchy of a particular user interface is available. The limitation of this approach is that it can only be employed to access interactive elements, such as icons and no other random locations or regions.

This paper proposes a new interaction technique for the motor disabled users using a switch based mechanism, aimed towards efficiency and easier access. 


\section{MATRIX SCAN}

Matrix Scan is a switch aided access mechanism for screen traversal, which divides the touch screen interface in a 2DMatrix and then further subdivides the regions into smaller cells, until the desired region can be conveniently distinguished. This division at differential levels is triggered and controlled by a simple physical movement on the external Bluetooth switch. The Matrix Scan access mechanism uses this switch to provide instructions to the device. The mechanism utilizes these instructions at each level in order to perform different levels of traversals to facilitate user in pointing and selecting a region on touch screen devices even with small movement ability. The fundamental concept behind this approach is that almost all the target views acquire fairly accessible region on the user interface, and can be easily selected by marking that area instead of pointing to a specific coordinate that falls inside the bounds of the desired view.

To interact with the Matrix Scan the user would require a Bluetooth switch. Switches are simple tool used to interact and issue commands to devices from a distance, usually paired over a Bluetooth connection. Conventional switches are capable of sending signals on some mechanical input by user, such as a simple tap, change in position or some other concise physical movement [9]. These switches can be operated using hands as well as feet. The fact that they provide the ability to generate instructions with minimal physical movement makes them an excellent choice to be used as an aid in access mechanism for motor disabled users.

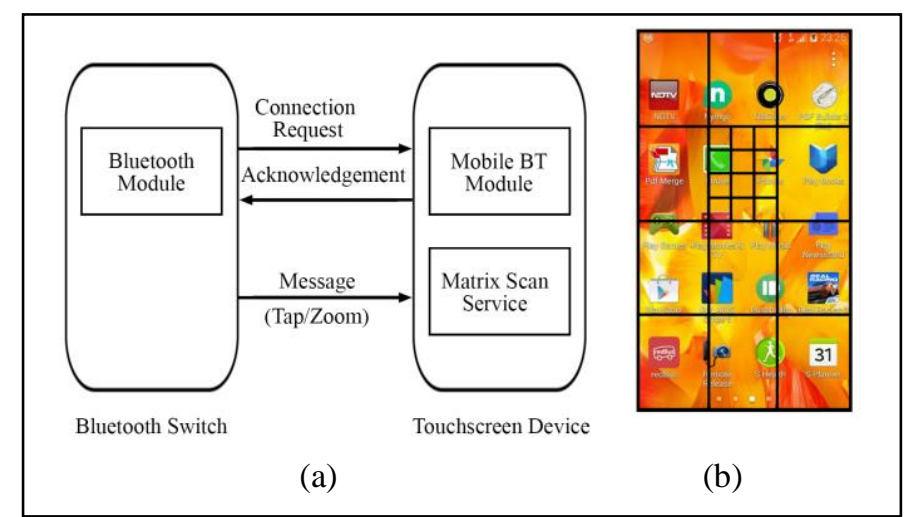

Fig 1(a): Architecture block diagram of the proposed solution. (b): A basic design of the proposed approach with matrix division of the touch area into two levels which can be applied into any touch screen device.

According to the study done by Dandekar K [10] the pixel width of the average index finger are 45-57 pixels which is higher than what mobile guidelines suggest. Similar study was done by Parhi, P [11] to calculate the average pixel width of thumb, which was concluded as around 72 pixels. But since mobile screens have a space constraint the guidelines suggest a smaller pixel width and height. For most of the modern devices there are guidelines for the minimum target size on the screen. Apple's iPhone human interface guidelines, Microsoft's Windows phone UI design and interaction guide and Nokia's developer guidelines suggest a minimum target size of $44 \mathrm{px} X$ $44 p x, 34 p x \times 34 p x$ and 28px X 28px respectively [12]. This is in accordance with Fitt's Law [13], which says that the time to reach a target is longer if the target is smaller. A small target slows users down because they have to pay extra attention to hit the target accurately. A finger-sized target gives users enough room to hit it without having to worry about accuracy [14].

\section{A. Method of Simulation}

In order to get real time stats for the aforementioned concept a sample application was developed on the Android platform which divides the screen in 2D matrices and delivers the touch once the desired view is reached. This was simulated on two devices, one device as a Bluetooth switch having two buttons tap and zoom; and the other device hosted the application for matrix based traversal.

The application hosts a service which draws lines to divide the screen in a $4 \times 3$ matrix. The cells formed are traversed automatically focussing each cell for a fixed interval. As the user presses the tap button on the BT switch a message is passed to the service and the cell focussed at that time would be selected and the touch can be delivered at the centre or corners of that cell. For more precision that selected cell could further be divided into $4 \times 4$ matrix providing much smaller cells which again automatically keep on traversing. These cells provide a small enough area to access most of the views. In the rarest of scenarios a further third level division of cells could be required which is also provided by the application. Also sometimes, user may miss to locate the correct cell in an attempt, which accounts to a failed attempt. Most of the times, in such cases the wrongly selected cell was either the cell previous or next to the intended cell. So in order to reduce time taken to locate the correct cell after a failed attempt, the traversal resumes from the cell previous to the last selected cell, such that the intended cell could be reached in less time upon resumption.

Samsung Galaxy Mega 6.3 device was used to host the application. And on reaching into the third level of matrix division the smallest block size of $2.9 \mathrm{~mm} \times 2.9 \mathrm{~mm}$ or $26.6 \mathrm{x}$ 26.6 pixels was achieved, which is sufficient to successfully identify a touch region on the basis of aforementioned studies [15].

To obtain a practical observation, following study was conducted on the most commonly used touch based interaction device i.e. smartphone. These observations were used to analyse the views and elements that are targeted in routine day to day usage of smartphones. This experimental study covered 280 views of which $49(17.5 \%)$ were covered within first matrix division which means a single click, 223(79.6\%) were covered in the second matrix division and $8(2.8 \%)$ were covered in the third matrix division amounting to three clicks.

TABLE 1: A STUDY ON NUMBER OF SWITCH CLICKS

\begin{tabular}{|c|c|}
\hline Number of clicks & Percentage of views reached $\mathbf{( \% )}$ \\
\hline 1 & 17.5 \\
\hline 2 & 79.6 \\
\hline 3 & 2.9 \\
\hline
\end{tabular}


In order to better understand Matrix Scan, conversations and interviews with both motor disabled people and also their doctors were conducted. These interactions were aimed at finding both the real issues faced by such user groups and how useful the proposed method could prove in solving them. The implementation of the method was provided to the participants and their respective feedbacks were recorded. One of the participants, a medical attendant from neurological department of a known medical institute, approved of this method and also suggested that it will be helpful for people suffering with Parkinson's disease and some cases of paralysis and spinal cord injuries. Another participant, a medical practitioner with experience of handling subjects with neurological disorder, was of the view that this method held significant value and that the design of the switch could add to the ease of the target users.

\section{FUTURE WORK}

There is much potential for future work in making touch screen devices more accessible for differently abled users. The initial study provided some encouraging results and a better perspective for designing a useful solution.

In this stage, the primary focus was to provide access to regular user interface elements like buttons, text boxes and widgets with precision and in least possible number of steps. The outcome was promising and the proposed approach was both consistent and reliable in most of the cases. In the followup study, we would like to explore the possibility of application of this approach to non-regular user interfaces. For example, in specific games, instead of common views, user may need to locate a certain point to perform an action. Another case that needs improvement is while typing through IME (Soft Input Panel), in which the error in precision was a lot higher than other cases. In order to provide support for such scenarios, it will be required to include methods in the proposed approach to handle such cases.

\section{CONCLUSION}

In this paper, a matrix based switch aided screen traversal mechanism to enhance accessibility of touch screen devices is proposed. A set of evaluations involving 12 disabled participants over different parameters demonstrated the ease and consistency in locating a desired element on user interface using the proposed method. During the evaluations, some major factors were identified with respect to motor disabled users, namely, ease of access, number of clicks required to select an item and comfort in learning the method, in which the proposed method was able to show significant improvements than existing methods. In majority cases, the intended element was locked in two steps, thus demanding minimal physical movement from user, which is essential considering limited motor abilities of user.
TABLE 2:USER EVALUATION OF METHOD OF SIMULATION FOR MATRIX SCAN. A SURVEY WAS CONDUCTED IN WHICH 12 MOTOR IMPAIRED PARTICIPANTS WERE ASKED TO RATE OUR METHOD IN DIFFERENT ASPECTS.

\begin{tabular}{|l|c|}
\hline Quality Metrics & $\begin{array}{l}\text { Average Score (out } \\
\text { of 5) }\end{array}$ \\
\hline Software is easy to use & 4.0 \\
\hline $\begin{array}{l}\text { It helped in making touch screen devices more } \\
\text { accessible }\end{array}$ & 3.75 \\
\hline $\begin{array}{l}\text { I was able to learn how to use this method without } \\
\text { much discomfort }\end{array}$ & 4.16 \\
\hline $\begin{array}{l}\text { I was able to navigate through the device with fair } \\
\text { ease }\end{array}$ & 4.0 \\
\hline The software is engaging & 4.41 \\
\hline
\end{tabular}

\section{REFERENCES}

[1] Bryen, D.N. and Pecunas, P. (2004) Augmentative and alternative communication and cell phone use: One off-the-shelf solution and some policy considerations. Assistive Technology, 16(1).11-17.

[2] Myers, B.A., Wobbrock, J.O., et. al. (2002) Using handhelds to help people with motor impairments. Proc. of ASSETS '02. Edinburgh, Scotland.pp.89-96.

[3] Olsen Jr, D.R. and Nielsen, T. "Laser Pointer Interaction," in ACM CHI'2001 Conference Proceedings: Human Factors in Computing Systems. 2001. Seattle, WA: pp. 17-22.

[4] Choi YS, Anderson CD, Glass JD, Kemp CC (2008) Laser pointers and a touch screen: Intuitive interfaces to an autonomous mobile robot for the motor impaired. In: ACM SIGACCESS Conference on Computers and Accessibility.

[5] Zhai, S., Morimoto, C., \& Ihde, S. (1999). Manual and gaze input cascaded (MAGIC) pointing.In: Proceedings of the ACM CHI'99 Human Factors in Computing Systems Conference (pp. 246-253). Addison-Wesley/ACM Press.

[6] Jacob, R. J. K. Eye movement-based human-computer interaction techniques: Toward non-command interfaces. In Advances in HumanComputer Interaction, Vol. 4, ed. H. R. Hartson and D. Hix, Ablex Publishing Co. (1993), Norwood, N.J., 151-190.

[7] J. Froehlich, J. O. Wobbrock, and S. K. Kane. Barrier pointing: using physical edges to assist target acquisition on mobile device touch screens. In ASSETS 2007, pages 19-26, 2007. ACM.

[8] Guerreiro, T., Nicolau, H., Jorge, J., \& Gonçalves, D. (2010). Towards Accessible Touch Interfaces, Proceedings of ACM SIGACCESS conference on Computers and Accessibility (ASSETS '10), pp. 19-26, ISBN 978-1-60558-881-0, Orlando, Florida, October 25-27, 2010.

[9] Tai,K., Blain, S. and Chau,T.(2008) A review of emerging access technologies for individuals with severe motor impairments. Assistive Technology: The Official Journal of RESNA.Volume 20, Issue 4, '08.

[10] Dandekar K, Raju BI, Srinivasan MA. 3-D finite-element models of human and monkey fingertips to investigate the mechanics of tactile sense. J Biomech Eng. 2003; 125:682-691. [PubMed](2003)

[11] Parhi, P., Karlson, A., Bederson, B. Target Size Study for One-Handed Thumb Use on Small Touchscreen Devices. Proc. MobileHCI'06. 203210. 2006

[12] Anthony, T. Finger-Friendly Design: Ideal Mobile Touchscreen Target Sizes: http://www.smashingmagazine.com/2012/02/21/finger-friendlydesign-ideal-mobile-touchscreen-target-sizes/. February 21st, 2012.

[13] MacKenzie, I. S. (1992). Fitts' law as a research and design tool in human-computer interaction. Human-Computer Interaction, 7, 91-139.

[14] Potter, R., Weldon, L., Shneiderman, B. Improving the Accuracy of Touchscreens: An Experimental Evaluation of Three Strategies. Proc. CHI'88. 27-32. 1988.

[15] Albinsson, P. and Zhai, S. 2003. High precision touch screen interaction. Proc. CHI'03. 105-112. 2003. 